\title{
GENERATIONS OF WAVES AT AN INERTIAL SURFACE DUE TO EXPLOSIONS
}

\author{
B.N. MANDAL and N.K. GHOSH
}

\author{
Department of Applied Mathematics \\ University of Calcutta \\ 92, A.P.C. Road \\ Calcutta - 700009 \\ India
}

(Received March 25, 1988)

\begin{abstract}
This paper is concerned with the generation of surface waves by an axially symmetric initial disturbance applied at an inertial surface in an ocean of finite depth. This initial disturbance can be efther in the form of an impulse or an elevation or depression. The depression of the inertial surface is obtained as an infinite integral in each case. The method of stationary phase is applied to evaluate the integral for large values of time and distance.
\end{abstract}

KEY WORDS AND PHRASES. Ocean of finite depth, surface distribution, inertial surface, velocity potential, Laplace transform, Hanke1 transform, method of stationary phase. 1980 AMS SUBJECT CLASSIFICATION CODE. 76B15.

\section{INTRODUCTION.}

Waves are generated by an explosion above or within an ocean. The formulation of the problems associated with the generation of these waves as an initial value problem is based on the linear theory of surface water waves. If the explosion occurs above the ocean surface, the initial condition on the surface is taken as an initial impulse distributed over a certain region while for the case when the explosion occurs at or below the ocean surface, the initial condition is taken as an initial elevation or depression of the same surface. Stoker [1] and Lamb [2] considered the two dimensional unsteady motion due to an initial surface disturbance concentrated at the origin and gave the velocity potential as well as the asymptotic form of the free surface for large values of time and distance. Kranzer and Keller [3] considered the three dimensional unsteady motion due to an arbitrary axially symmetric initial surface disturbance in an ocean of uniform finite depth and gave explicit formula for the surface elevation and compared the theory with experimental results. They also deduced these results for an ocean of infinite depth. Chaudhuri [4] and Wen [5] considered the case when the initial surface disturbance is a combination of both surface impulse and surface elevation distributed over an arbitrary region of the 
surface of an ocean of uniform finite depth and obtained asymptotic results by using the method of stationary phase.

In all these cases, the ocean was essentially bounded above by a free surface. Recently there has been a considerable interest in various problems concerned with the generation of surface waves due to different types of sources of arbitrary timedependent strengths present in a liquid covered by an inertial surface composed of a thin but uniform distribution of noninteracting floating particles (e.g. broken ice floating on an ocean surface) (see Rhodes-Robinson [6], Mandal and Kundu [7,8] and Manda1 [9,10]). It is thus natural to extend the problems due to initial surface disturbances in an ocean with a free surface to the case when the ocean is covered by an inertial surface. Recently, Mandal [10] considered the two dimensional unsteady motion in a deep ocean covered by an inertial surface due to initial disturbances at the inertial surface while Mandal and Mukherjee [11] considered the three dimensional unsteady motion due to a prescribed axisymmetic initial surface disturbance.

In this paper we extend the problem considered in [11] for a deep ocean to an ocean of uniform finite depth. This may also be viewed as an extension of the problem of generation of water waves at the free surface of an ocean of finite depth considered by Kranzer and Keller [3] to an ocean covered by an inertial surface. After formulating it within the framework of linearized theory as an initial value problem, it is reduced to a boundary value problem by taking the Laplace transform in time. The Hankel transform is then used to solve this boundary value problem. Finally, Laplace inversion gives the potential function from which the depression of the inertial surface is obtained in terms of an infinite double integral. By applying the method of stationary phase twice in succession to this integral, the asymptotic form of the inertial surface is obtained for large values of time and distance. In the absence of the parameter characterizing the inertial surface, known results given earlier in Kranzer and Keller [3] are recovered.

\section{STATEMENT AND FORMULATION OF THE PROBLEM.}

We consider the unsteady three dimensional motion of an inviscid, incompressible, homogeneous liquid of volume density $\rho$ covered by an inertial surface composed of thin but uniformly distributed disconnected floating matter of area density

$\rho \varepsilon(\varepsilon>0)$ under the action of gravity $g$ only. The liquid is of uniform finite depth h. $\varepsilon=0$ corresponds to a liquid with a free surface. We assume that the motion in the liquid is started at time $t=0$ from a state of rest by a prescribed axisymmetric inftial disturbance at the inertial surface. We choose a cylindrical polar coordinate system $(r, \theta, y)$ in which the $y$ axis is taken vertically downwards and is the axis of symmetry of the initial disturbance, the plane $y=0$ is the position of the inertial surface at rest, and $y=h$ is the bottom of the unlimited mass of the liquid. Since the motion starts from rest, it is irrotational and can be described by a velocity potential $\varphi(r, y ; t)$ for $t>0$. Within the framework of linearized theory, 
it can be shown that $\varphi$ satisfies

$$
\frac{1}{r} \frac{\partial \varphi}{\partial r}\left(r \frac{\partial \varphi}{\partial r}\right)+\frac{\partial^{2} \varphi}{\partial y^{2}}=0,0<y<h, t>0
$$

with the inertial surface condition

$$
\frac{\partial^{2}}{\partial t^{2}}\left(\varphi-\varepsilon \varphi_{y}\right)-g \varphi_{y}=0 \text { on } y=0, t>0
$$

while the boundary condition at the bottom is

$$
\varphi_{\mathrm{y}}=0 \text { on } \mathrm{y}=\mathrm{h}, \mathrm{t}>0
$$

If the initial disturbance is due to an axisymmetric displacement $G(r)$ of the inertial surface at a distance $r$ from the origin, then the initial conditions are

$$
\begin{aligned}
& \varphi-\varepsilon \varphi_{\mathrm{y}}=0, \mathrm{y}=0, \mathrm{t}=0, \\
& \frac{\partial}{\partial t}\left(-\varepsilon \phi_{\mathrm{y}}\right)=\mathrm{g} G(\mathrm{r}), \mathrm{y}=0, \mathrm{t}=0 .
\end{aligned}
$$

We note that the initial displacement of the inertial surface may be a depression or elevation depending on $G(r)$ being positive or negative. However, if the initial disturbance is due to an axially symmetric impulse of strength $F(r)$ at a distance $r$ from the origin, then the initial conditions are

$$
\begin{aligned}
& \varphi-\varepsilon \varphi_{\mathrm{y}}=-\frac{F(\mathrm{r})}{\rho} \text { on } \mathrm{y}=0, \mathrm{t}=0 \\
& \frac{\partial}{\partial t}\left(\varphi-\varepsilon \varphi_{\mathrm{y}}\right)=0 \text { on } \mathrm{y}=0, \mathrm{t}=0
\end{aligned}
$$

3. SOLUTION OF THE PROBLEM.

Let $\Phi(r, y ; p)$ denote the Laplace transform of $\varphi(r, y ; t)$ in time $t$ defined as

$$
\Phi(r, y ; p)=\int_{0}^{\infty} \varphi(r, y ; t) e^{-p t} d t, p>0 .
$$

Then $\Phi(r, y ; p)$ satisfies the boundary value problem

$$
\begin{aligned}
& \frac{1}{r} \frac{\partial}{\partial r}\left(r \frac{\partial \Phi}{\partial r}\right)+\frac{\partial^{2} \Phi}{\partial y^{2}}=0,0<y<h, \\
& p^{2} \Phi-\left(g+\varepsilon p^{2}\right) \Phi_{y}=\left\{\begin{array}{c}
g G(r) \text { on } y=0 \\
\text { or } \\
-\frac{p}{\rho} F(r) \text { on } y=0
\end{array}\right.
\end{aligned}
$$

and $\Phi_{y}=0$ on $y=h$. 
(3.2a) holds for an initial surface displacement while (3.2b) holds for an initial surface impulse. Let $\Psi(k ; y)$ be the Hankel transform of $\Phi(r ; y)$ defined as

$$
\Psi(k ; y)=\int_{0}^{\infty} r \Phi(r ; v) J_{0}(k r) d r, k>0
$$

Then $\Psi$ satisfies

$$
\begin{aligned}
& \frac{d^{2} \Psi}{d y^{2}}-k^{2} \Psi=0,0 \leqslant y \leqslant h, \\
& p^{2} \Psi-\left(g+\varepsilon p^{2}\right) \Psi_{y}=\left\{\begin{array}{l}
g \hat{G}(k) \\
\text { or } \\
-\frac{p}{\rho} \hat{F}(k)
\end{array}\right.
\end{aligned}
$$

and

$$
\Psi_{y}=0 \text { on } y=h \text {. }
$$

Here $\hat{G}(k)$ and $\hat{F}(k)$ are the Hankel transforms of $G(r)$ and $F(r)$ respectively.

Then

$$
\Psi(k, y)=\left\{\begin{array}{c}
g \hat{G}(k) \frac{\operatorname{coshk}(h-y)}{p^{2} D(k)+g k \sinh k h} \\
o r \\
-\frac{p}{\rho} \hat{F}(k) \frac{\operatorname{coshk}(h-y)}{p^{2} D(k)+g k \sinh k h}
\end{array}\right.
$$

where $D(k)=\cosh k h+\varepsilon k \sinh k h$.

Hence

where $\mu^{2}=\frac{g k \sinh k h}{D(k)}$.

$$
\Phi(r, y ; p)=\left\{\begin{array}{c}
g \int_{0}^{\infty} \frac{\hat{G}(k) k \cosh k(h-y) J_{0}(k r)}{D(k)\left(p^{2}+\mu^{2}\right)} d k \\
o r \\
-\frac{1}{\rho} \int_{0}^{\infty} \frac{p k \hat{F}(k) \cosh k(h-y) J_{0}(k r)}{D(k)\left(p^{2}+\mu^{2}\right)} d k
\end{array}\right.
$$

Taking the Laplace inversion we obtain the potential function. For the case of an initial axially symmetric displacement we use (3.10a) to obtain

$$
\varphi(r, y ; t)=\int_{0}^{\infty} \frac{\hat{G}(k) J_{0}(k r) \mu \sin \mu t \operatorname{coshk}(h-y)}{\text { sinhkh }} d k \text {. }
$$


For the case of initial axially symmetric impulse we obtain from (3.10b)

$$
\varphi(r, y ; t)=-\frac{1}{\rho g} \int_{0}^{\infty} \frac{\hat{F}(k) \mu^{2} \cos \mu t \operatorname{coshk}(h-y) J_{0}(k r)}{\operatorname{sinhkh}} d k \text {. }
$$

In these cases the inertial surface depression $\xi(r, t)$ is given by

$$
\xi(r, t)=\frac{1}{g}\left[\frac{\partial}{\partial t}\left(\varphi-\varepsilon \varphi_{y}\right)\right]_{y=0}
$$

so that

$$
\xi(r, t)=\left\{\begin{array}{c}
\int_{0}^{\infty} k \hat{G}(k) J_{0}(k r) \cos \mu t d k \\
\text { or } \\
\frac{1}{\rho g} \int_{0}^{\infty} k \hat{F}(k) \mu \sin \mu t J_{0}(k r) d k .
\end{array}\right.
$$

The results given in Kranzer and Keller [3] are recovered by making $\varepsilon=0$. Also, by making $h \rightarrow \infty$ and setting $\varepsilon=0$, results given in Stoker [1] are recovered.

\section{ASYMPTOTIC EXPANSIONS.}

The method of stationary phase is now applied to obtain an asymptotic form of $\xi(r, t)$ for the integral in equation (3.12a) for large $t$ and $r$ but finite ratio $r / t$. Using the result

$$
J_{0}(k r)=\frac{2}{\pi} \int_{0}^{\pi / 2} \cos (k r \cos \beta) d B
$$

(3.12a) is written as

$$
\begin{aligned}
\xi(r, t) & =\frac{1}{\pi} \operatorname{Re} \int_{0}^{\infty} \int_{0}^{\pi / 2} k \hat{G}(k)\left[\exp \left\{1 t\left(\mu+k \frac{r}{t} \cos \beta\right)\right\}\right. \\
& \left.+\exp \left\{1 t\left(\mu-k \frac{r}{t} \cos \beta\right)\right\}\right] d \beta d k .
\end{aligned}
$$

By using the method of stationary phase first to the B-integral, we get

$$
\xi(r, t) \simeq\left(\frac{2 \pi}{r}\right)^{\frac{1}{2}} \int_{0}^{\infty} k^{\frac{1}{2}} \hat{G}(k)\left[\exp \left\{1 t P_{1}(k)\right\}+\exp \left\{1 t P_{2}(k)\right\}\right] d k
$$

$$
\begin{array}{ll}
\text { where } & P_{1}(k)=\mu+\frac{k r}{t}-\frac{\pi}{4 t} \\
\text { and } & P_{2}(k)=\mu-\frac{k r}{t}+\frac{\pi}{4 t} . \\
\text { Now } & P_{2}^{\prime}(k)=Q(k)-\frac{r}{t}
\end{array}
$$


where $Q(k)=\frac{1}{2}(g h)^{1 / 2}\left[\frac{1}{\{D(k)\}^{3 / 2}}\left\{\left(\frac{k h}{\sinh k h}\right)^{1 / 2}+\cosh k h\left(\frac{s i n h k h}{k h}\right)^{1 / 2}\right\}\right]$

so that $P_{2}^{\prime \prime}(k)=Q^{\prime}(k)$.

It is obvious that $P_{2}^{\prime \prime}(k)<0$ for $0<k<\infty_{0}$ Hence $P_{2}^{\prime}(k)$ is a strictly monotonic decreasing function in $0<k<\infty$. Also

$$
\mathrm{P}_{2}^{\prime}(\mathrm{k})+(\mathrm{gh})^{1 / 2}-\frac{\mathrm{r}}{\mathrm{t}} \text { as } \mathrm{k}+0^{+}
$$

and

$$
\mathrm{P}_{2}^{\prime}(\mathrm{k}) \rightarrow-\frac{\mathrm{r}}{\mathrm{t}} \text { as } \mathrm{k} \rightarrow \infty
$$

These show that there is no stationary point in the integral for $r / t>(g h)^{1 / 2}$ while for $r / t<(g h)^{1 / 2}$, there is a single stationary point in $0<k<\infty$ so that there exists only one stationary point at $k=\alpha$ (say), where $\alpha$ is the real positive root of the equation $P_{2}^{\prime}(k)=0$. When $r / t=(g h)^{1 / 2}$, it gives a smaller contribution than the $r / t<(g h)^{1 / 2}$ case so that contribution from it may be neglected. Also $P_{1}(k)$ has no stationary point in the range of integration. The stationary phase method for the $k$ integral when applied to (4.2) leads to

$$
\begin{aligned}
\xi(r, t) & \simeq \frac{\hat{G}(\alpha)}{r^{1 / 2}}\left(\frac{\alpha}{t\left|P^{\prime \prime}(\alpha)\right|}\right)^{1 / 2} \cos \left(t P_{2}(\alpha)-\frac{\pi}{4}\right) \\
& =\frac{\hat{G}(\alpha)}{r}\left(\frac{\alpha Q(\alpha)}{-Q^{\prime}(\alpha)}\right)^{1 / 2} \cos (\mu(\alpha) t-\alpha r)
\end{aligned}
$$

where

$$
\mu(\alpha)=\left(\frac{g \alpha \tanh \alpha h}{1+\varepsilon \alpha \tanh \alpha h}\right)^{1 / 2}
$$

In particular, when the displacement is concentrated at the origin, $\hat{G}=\frac{1}{2 \pi}$. In this case

$$
\xi(r, t) \simeq \frac{1}{2 \pi r}\left(\frac{\alpha Q(\alpha)}{-Q^{\prime}(\alpha)}\right)^{1 / 2} \cos (\mu(\alpha) t-\alpha r)
$$

Without solving for $\alpha$ from the equation $\mathrm{P}_{2}^{\prime}(\alpha)=0$, we consider the following two special cases.

CASE 1. Shallow ocean (h small).

For small values of $h$, we can approximate $\mu$ as

$$
\mu \simeq\left[g k^{2} h\left(1-\frac{1}{3} k^{2} h^{2}-\varepsilon k^{2} h\right)\right]^{1 / 2}
$$


so that $\quad \alpha=\left[\frac{3\left\{g h-\left(\frac{r}{t}\right)^{2}\right\}}{h^{2}\left\{4 g h+9 g-\left(\frac{r}{t}\right)^{2}\right\}}\right]^{1 / 2}$,

$$
\begin{aligned}
P^{\prime \prime}(\alpha)= & Q^{\prime}(\alpha)=\frac{(\mathrm{gh})}{\left\{\alpha^{2}\left(1-\frac{1}{3} \alpha^{2} h^{2}\right)\right\}^{3 / 2}}\left[\alpha^{2}\left(1-\frac{1}{3} \alpha^{2} h^{2}\right)\left\{1-\frac{1}{2} \alpha^{2} h(9 \varepsilon+4 h)\right\}\right. \\
& \left.-\alpha^{2}\left(1-\frac{2}{3} \alpha^{2} h^{2}\right)\left\{1-\frac{1}{6} \alpha^{2} h(9 \varepsilon+4 h)\right\}\right] .
\end{aligned}
$$

Using (4.8) and (4.9) in (4.5) we obtain $\xi(r, t)$.

The asymptotic form of the inertial surface given in (4.5) can also be expressed in the form

$$
\xi(r, t) \simeq \frac{\hat{G}(\alpha)}{r}\left(\frac{\alpha Q(\alpha)}{-Q^{\prime}(\alpha)}\right)^{1 / 2} \cos \left[2 \pi\left(\frac{t}{T}-\frac{r}{\lambda}\right)\right]
$$

where

$$
T=\frac{2 \pi}{\mu(\alpha)} \text { and } \lambda=\frac{2 \pi}{\alpha} \text {. }
$$

For the case of an initial axially symmetric displacement, we obtain by a similar analysis the asymptotic form $\xi(r, t)$ of the inertial surface given by

$$
\begin{aligned}
\xi(r, t) & \simeq \frac{1}{\rho(r g)^{1 / 2}} \frac{\alpha \hat{F}(\alpha)}{(1+\varepsilon \alpha \tanh \alpha)^{1 / 2}}\left(\frac{\tanh \alpha h}{\left.t \mid P^{\prime \prime}(\alpha)\right)^{1 / 2}} \sin (\mu(\alpha) t-\alpha r)\right. \\
& =\frac{1}{\rho g^{1 / 2} r} \frac{\alpha \hat{F}(\alpha)}{(1+\varepsilon \alpha \tanh \alpha)^{1 / 2}}\left(\frac{Q(\alpha) \tanh \alpha h}{-Q^{\prime}(\alpha)}\right)^{1 / 2} \sin \left[2 \pi\left(\frac{t}{T}-\frac{r}{\lambda}\right)\right]
\end{aligned}
$$

where $\alpha$ is given by $(4.8)$ and $Q^{\prime}(\alpha)$ is given by (4.9).

When the impulse is concentrated at the origin $\hat{F}=\frac{1}{2 \pi}$. In this case, the asymptotic form of the inertial surface has the form given by

$$
\xi(r, t)=\frac{\alpha}{2 \pi \rho g^{1 / 2}}\left(1-\frac{1}{2} \varepsilon \alpha^{2} h\right)\left(\frac{Q(\alpha) \tanh \alpha h}{-Q^{\prime}(\alpha)}\right)^{1 / 2} \sin \left[2 \pi\left(\frac{t}{T}-\frac{r}{\lambda}\right)\right] \quad \text { (4.13) }
$$

The result given in (4.5) and (4.12) are in complete agreement with the corresponding results given by Kranzer and Keller [3] if we put $\varepsilon=0$ and $\alpha=\frac{\sigma}{h}$.

CASE II. Deep ocean (h is infinite)

$$
\mu=\left(\frac{g k}{1+\varepsilon k}\right)^{1 / 2} \text { and from the equation } P_{2}^{\prime}(k)=0
$$

we get $k^{1 / 2}(1+\varepsilon k)^{3 / 2}=\frac{g^{1 / 2} t}{2 r}$. 
In this case the asymptotic form of the inertial surface can be obtained by a 8 imilar analysis and the corresponding results given by Mandal and Mukherjee [11] are easily recovered.

5. DISCUSSION.

From equation (4.10) and (4.12) we see that the surface wave amplitude, the period $T$, and the wavelength $\lambda$ all depend upon $r /(g h)^{1 / 2} t$ as well as $\varepsilon_{\text {. The amplitude }}$ in both cases is zero if $r /(\mathrm{gh})^{1 / 2} \mathrm{t}>1$. The factor $\sin 2 \pi[t / T-r / \lambda]$ or $\cos 2 \pi[t / T-r / \lambda]$ describes the actual waves. If $r=(g h)^{1 / 2}$, both the wavelength and period will be infinite in wave motion and both decrease for fixed $t$ or as $t$ increases for fixed $r$. These observations were also arrived at for the corresponding problem in water with a free surface (cf [3]). Thus the qualitative behavior of the solutions remains unaltered due to the presence of an inertial surface.

\section{REFERENCES}

1. STOKER, J.J., Water waves, Interscience, New York, 1957.

2. LAMB, H., Hydrodynamics, Dover, New York, 1945.

3. KRANZER, H.C. and KELLER, J.B., Water waves produced by explosion, J. App1. Phys. 30 (1959), 398-407.

4. CHAUDHURI, K., Waves in shallow water due to arbitrary surface disturbances, App1. Sc1. Res. 19 (1968), 274-284.

5. WEN, S.L., A note on water waves created by surface disturbances. Int. J. Math. Edu. Sc1. Techno1. 13 (1982).

6. RHODES-ROBINSON, P.F., On the generation of water waves at an inertial surface. J. Austral. Math. Soc. Ser. B.25 (1984), 366-383.

7. MANDAL, B.N. and KUNDU, K., A note on the singularities in the theory of water waves with an inertial surface. J. Austra1. Math. Soc. Ser B. 28 (1986), 271278.

8. MANDAL, B.N., Ring source potential in a liquid with an inertial surface Mech. Res. Comm. 13 (1986), 335-339.

9. MANDAL, B.N. and KUNDU, K., Ring source potential in a 1iquid with an Inertial surface in the presence of surface tension, Int. J. Engg. Sc1. 25 (1987).

10. MANDAL, B.N., Water waves generated by disturbance at an interial surface. App1. Sc1. Res., (1987).

11. MANDAL, B.N. and MUKHERJEE, S., Water waves generated at an inertial surface by an axisymmetric initial surface distribution. Int. J. Math. Edu. Sc1. Techno1., (1987, accepted). 


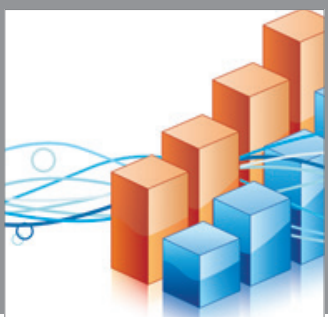

Advances in

Operations Research

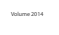

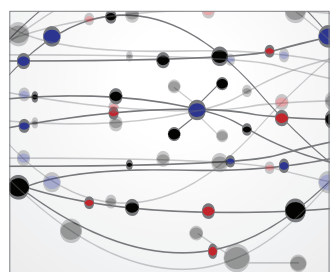

\section{The Scientific} World Journal
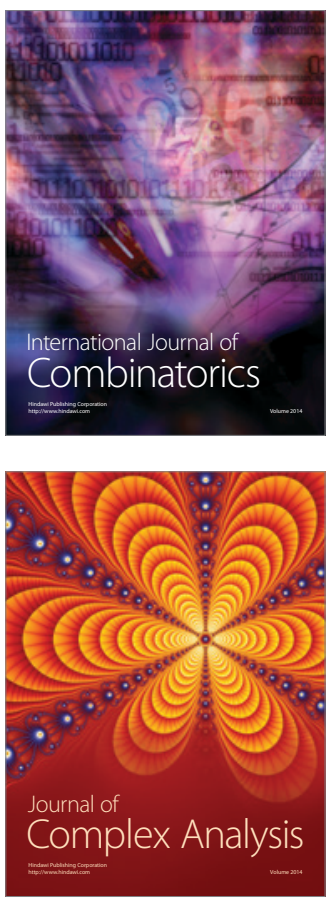

International Journal of

Mathematics and

Mathematical

Sciences
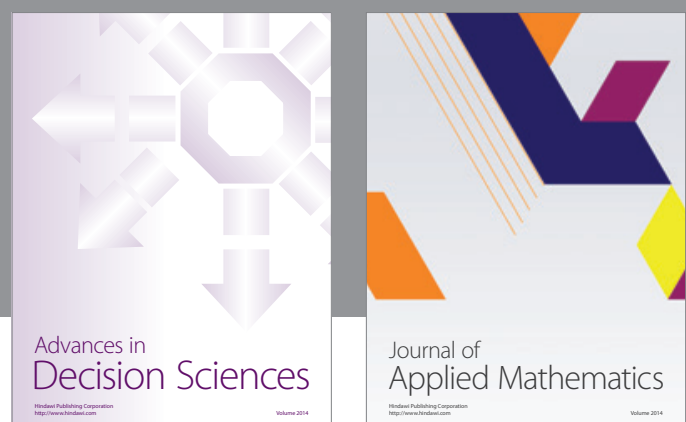

Journal of

Applied Mathematics
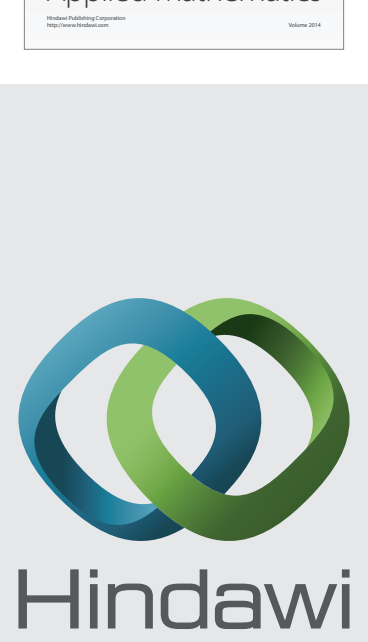

Submit your manuscripts at http://www.hindawi.com
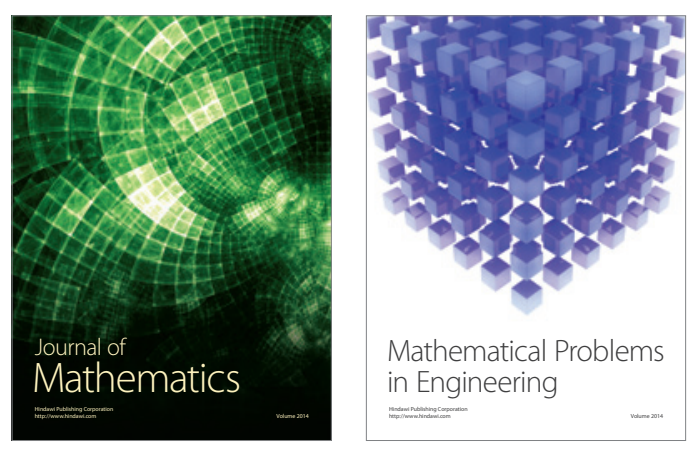

Mathematical Problems in Engineering
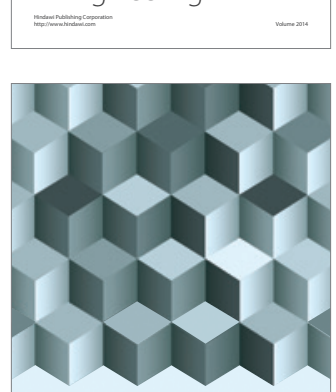

Journal of

Function Spaces
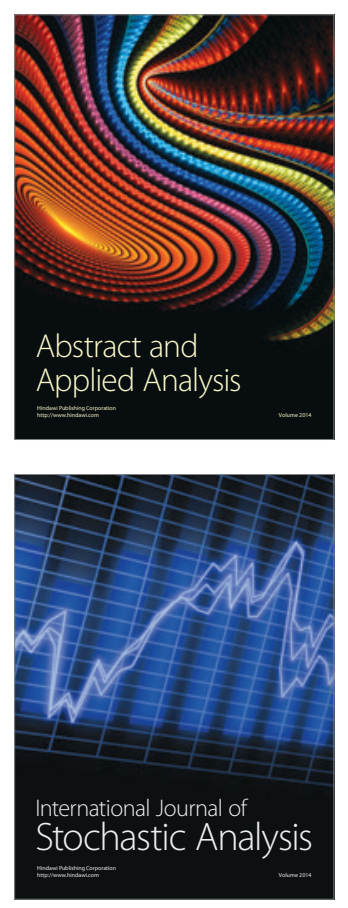

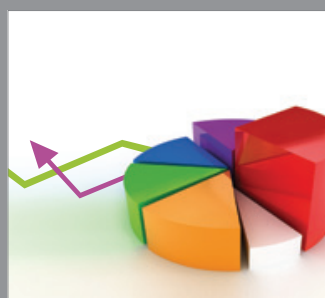

ournal of

Probability and Statistics

Promensencen
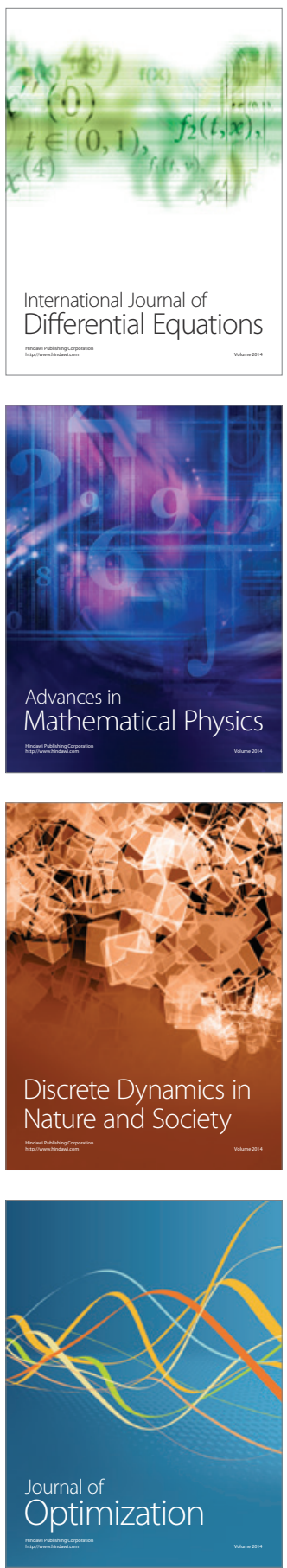